\title{
$f$-band condensates in exciton-polariton lattice systems
}

\author{
Na Young Kim, ${ }^{1, *}$ Kenichiro Kusudo, ${ }^{2,3}$ Andreas Löffler, ${ }^{4}$ Sven Höfling, ${ }^{4}$ Alfred Forchel,${ }^{4}$ and Yoshihisa Yamamoto ${ }^{1,2}$ \\ ${ }^{1}$ E. L. Ginzton Laboratory, Stanford University, 348 Via Pueblo Mall, Stanford, California, 94305 USA \\ ${ }^{2}$ National Institute of Informatics, 2-1-2 Hitotsubashi, Chiyoda-ku, Tokyo 101-8430, Japan \\ ${ }^{3}$ NTT Basic Research Laboratories, 3-1 Morinosata-Wakamiya, Atsugi 243-0198, Japan \\ ${ }^{4}$ Technische Physik and Wilhelm-Conrad-Röntgen-Research Center for Complex Material Systems, Universität Würzburg, \\ D-97074 Würzburg, Am Hubland, Germany
}

(Received 7 March 2013; revised manuscript received 4 December 2013; published 12 February 2014)

\begin{abstract}
We report the condensation of microcavity exciton-polaritons at $\Gamma$ points located on the boundary between the third and higher Brillouin zones in hexagonal lattices: triangular and honeycomb geometries. We collect experimental evidence that supports the finite momentum condensation: (1) the coherent Bragg peaks formed at nonzero $\Gamma$ points; (2) the nonlinear intensity increase in the exciton-polariton emission at quantum degeneracy threshold; (3) the spectral linewidth behavior: narrowing near threshold and broadening above threshold; and (4) the equivalent $4 f_{y^{3}-3 x^{2} y}$-like orbital symmetry in real space. The $f$-orbital state at $\Gamma$ points appears as a metastable momentum valley to trap exciton-polaritons, which is explained by single-particle band-structure calculations.
\end{abstract}

DOI: 10.1103/PhysRevB.89.085306

\section{INTRODUCTION}

Quantum-mechanical particle-wave duality leads to the concept of matter waves (or de Broglie waves), which can preserve coherence in macroscopic scales and, consequently, interfere themselves. Coherent matter waves can be used from precision standards for metrology and sensing applications to the basis for the investigation of fundamental physical properties. Among several sources to produce coherent beams with massive particles, atomic Bose-Einstein condensates have attracted special attention in that they can be formed and manipulated in a delicate and reproducible manner. In particular, zero-momentum atomic condensates in optical lattices have been a successful platform for exploring condensed matter physics in a photonic domain [1] since the seminal work that reported the superfluid-Mott insulating quantum phase transition governed by a simplest Bose-Hubbard Hamiltonian [2].

Despite the impressive progress, there is a primary hindrance of the early bosonic atom-lattice system in simulating many-body collective behavior of strongly correlated materials: the predominant ground-state $s$-wave orbital symmetry of boson condensates. According to the no-node theorem, R. P. Feynman showed that the conventional ground state wave function of short-ranged interacting bosons, without external rotation, is nondegenerate and positive definite in the coordinate space $[3,4]$. Its unique bosonic property does not share the common grounds with high-orbital fermionic features emerging in strongly correlated materials [5]. With this very reason, a serious effort has been made in searching for exotic emergent states of bosons beyond the no-node theorem, which would provide the complementary picture to the fermionic many-body physics. An approach is to prepare coherent atoms at metastable states with multi-flavored orbital symmetry, where complex-valued many-body wave functions can occur. Theorists have proposed schemes to identify quantum phase orders in multi-flavor atomic condensates

\footnotetext{
*nayoung@stanford.edu
}

PACS number(s): 71.36.+c, 78.55.Cr, 78.67.De

in lattices [6-9]. Experimentalists have pursued to excite atomic condensates into $p$-wave $[10,11]$ and $f$-wave [12] symmetry orbital states via sophisticated population swapping techniques.

Microcavity exciton-polariton condensates are another form of coherent matter waves based on semiconductors [13]. The inherent dynamical nature makes them to readily access nonzero momentum states resulting from the interplay between the finite lifetime and the relaxation scattering rates. Recently, there are many attempts to engineer one- and two-dimensional (2D) exciton-polariton-lattice systems using various methods: acoustic waves [14], pillar-arrays [15,16], and thin-film techniques [17]. Using the thin metal-film deposition, we have demonstrated both metastable $p$ [18] and $d$ [19] condensations in a one-dimensional array and a 2D square lattice, respectively. Here, we are able to form the $f$-orbital condensation of exciton-polaritons in triangularsymmetric lattices, which may be a prelude to the study of emergent quantum phase orders associated with high orbital symmetry in 2D. Knowing the fact that the $d$ - and $f$-orbital electrons would be primarily responsible for richness of quantum phases exhibiting a variety of magnetic orders in transition oxides $[5,20]$, we anticipate to study $f$-orbital exciton-polariton condensates with spin degrees of freedom, which would provide important clues towards those materials.

\section{EXPERIMENT AND DISCUSSION}

Microcavity exciton-polaritons are hybrid light-matter quantum quasiparticles appearing typically in a monolithic quantum well-microcavity structure [21]. In the low density at low temperatures, exciton-polaritons are described by the linear superposition of two bosonic modes: quantum-well (QW) excitons and cavity photons, whose interaction coupling strength is called as vacuum Rabi splitting. Obeying the BoseEinstein statistics, exciton-polaritons undergo quantum phase transition from thermal particles to macroscopic condensates via stimulated scattering induced by exchange interactions above quantum degeneracy threshold [22-24]. The nonlinear 
increase of population in the condensate state and spontaneous buildup in spatial and temporal coherence are observed as associated signatures of bosonic condensation. Unlike atomic condensates, exciton-polariton condensates are naturally in a nonequilibrium condition because they leak from the cavity as photons so that they are continuously fed by an external pumping source. Hence the metastable condensates at finite momentum values are the outcome of the balance between the exciton-polariton decay rate and the stimulated scattering rate. In addition, we introduce $2 \mathrm{D}$ in-plane potentials using a simple metal-film deposition technique in order to manipulate the dynamics of exciton-polariton condensates via bandstructure engineering [17].

\section{A. Device and experimental setup}

The wafer under study consists of a multi-QW microcavity structure made of GaAs/AlAs semiconductors, whose detailed structure has been given in previous works $[18,19]$. On the top surface of the grown wafer, we deposit the thin metal-film of 2D triangular and honeycomb patterns as the array of circular apertures, whose lithographic center-to-center distance $(a)$ is set to be $4 \mu \mathrm{m}$ for the triangular and $2 \mu \mathrm{m}$ for the honeycomb lattices [see Figs. 1(a) and 1(b)]. Under the 23/5-nm Ti/Au metal film, the induced periodic potential strength is roughly 200-300 $\mu \mathrm{eV}$ depending on the fraction of photon modes because the metal film induces the blue-shifted cavity photon energy by $\sim 400 \mu \mathrm{eV}$. The total device dimension is $100 \mu \mathrm{m}$ by $100 \mu \mathrm{m}$, and the devices of interest are located in the wafer at the detuning values of $\Delta \sim-4.4 \mathrm{meV}$ for the triangular and $\sim-4.9 \mathrm{meV}$ for the honeycomb lattices. $\Delta$ quantifies the energy difference between the cavity photon energy and the QW exciton energy at zero momentum, and the negative value means that exciton-polaritons encounter more photonlike dispersion relations. Quantitatively, photon fractions are $65 \%$ and $67 \%$ for two aforementioned detuning values. Thus exciton-polaritons have a propensity to exhibit the bottleneck effect at large momentum values in which exciton-polaritons experience slower relaxation rates into the lower momentum and energy states due to the steeper curvature of the lower-polariton (LP) dispersions. This feature is in fact favorable to stabilize condensates with high orbital symmetry at finite momentum values when the dispersion is modified by the periodic potentials.

By means of microphotoluminescence imaging and spectroscopy in both real and momentum spaces at $\sim 4 \mathrm{~K}$, we characterize the distributions of exciton-polariton condensates under the 2D hexagonal lattices by exciton-resonant pulsed laser pumping at $\sim 60^{\circ}$ similar to the previous work [19]. The focused laser spot size is roughly $40 \mu \mathrm{m}$ by $30 \mu \mathrm{m}$, covering $\sim 10$ by 8 triangle unit cells in the triangular device and the 13 by 8 rhombus unit cells in the honeycomb device.

\section{B. Photoluminescence imaging and spectroscopy in momentum space}

Figures 1(c) and 1(d) depict the first and six higher-order Brillouin zones (BZs) specified with numbers 1-7 in the reciprocal space of triangular and honeycomb crystals, where
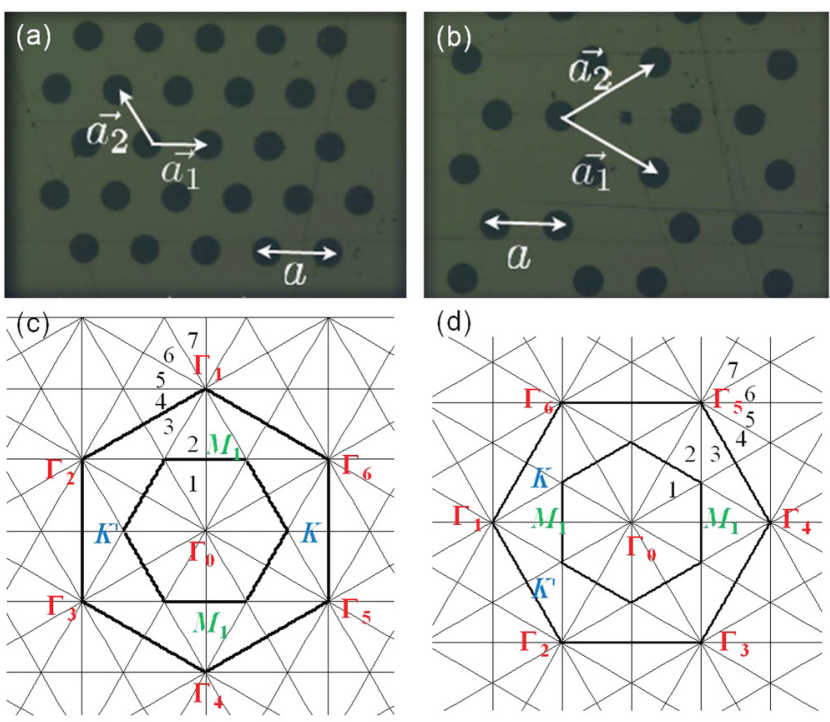

(d)
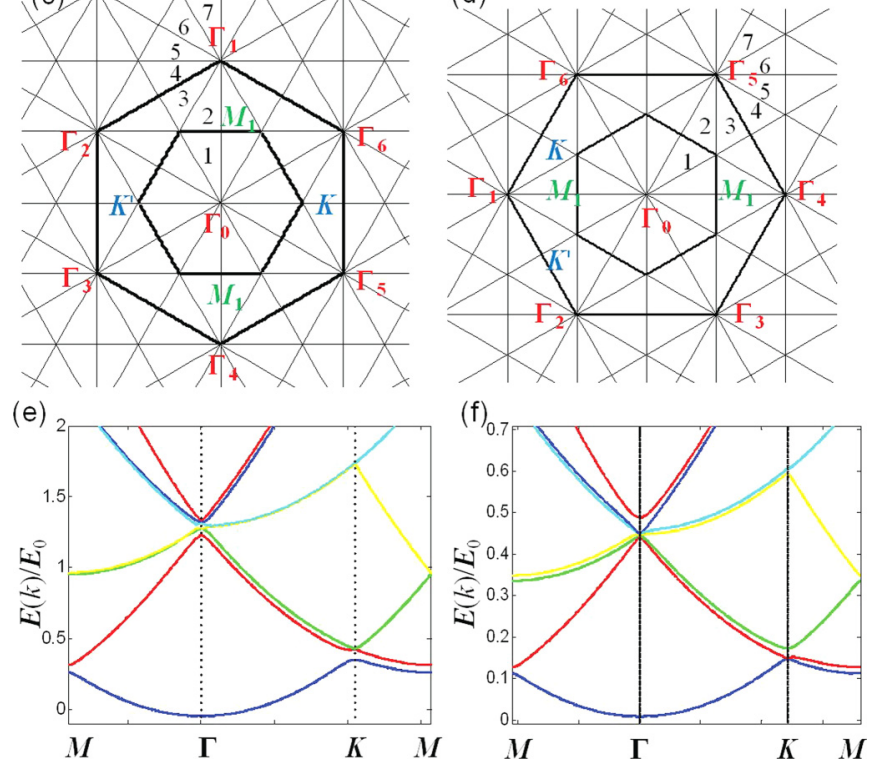

FIG. 1. (Color online) Photograph of a triangular (a) and a honeycomb (b) patterned devices, where $a$ is the center-to-center distance between the nearest-neighbor circular holes. The real-space unit vectors $\vec{a}_{1}$ and $\vec{a}_{2}$ are specified as basis for single-particle bandstructure calculations. The brighter area is where the thin metal film is deposited, and the darker circular zones show bare semiconductor surface. Two-dimensional extended Brillouin zones (BZs) of the triangular (c) and the honeycomb (b) lattices are presented with denotation of high-symmetry indices, $\Gamma_{i}(i=0-6), M, K$, and $K^{\prime}$. The first and third hexagonal BZs are emphasized by thick straight lines. The regions of the first seven BZs are numbered from 1 to 7 . The theoretical band structures of the triangular (e) and the honeycomb (f) lattices along the high-symmetry points is presented in the reduced zone scheme with the potential strength $V_{0}$ is $0.3 E_{0}$, where $E_{0}$ is the energy scaling unit for $a=4 \mu \mathrm{m}$ for the triangular and $a=2 \mu \mathrm{m}$ for the honeycomb lattices.

high-symmetry points are denoted as $\Gamma, M, K$, and $K^{\prime}$ with appropriate indices. The sixfold rotational degeneracy is clearly seen in $\Gamma_{i}$ points $(i=1-6)$ at the vertices of the third hexagonal BZs. The potential energy is weak, $20 \%-30 \%$ of the characteristic kinetic energy $E_{0}=\hbar^{2} / 2 m^{*}(2 \pi / a)^{2} \sim 1$ or $4 \mathrm{meV}$ for $a=4$ or $2 \mu \mathrm{m}$ with the effective mass, $m^{*} \sim$ $8 \times 10^{-5} m_{0}\left(m_{0}\right.$ is the bare electron mass), where $\hbar$ is the Planck constant divided by $2 \pi$. Hence, we treat the system in a single-particle picture and compute the band structure in terms of the plane wave basis (nearly-free single-particle approximation) $[25,26]$. The theoretical band structures are plotted in the reduced zone scheme along the high-symmetry points, $\Gamma, M, K$ in Figs. 1(e) and 1(f). Degenerate energy states are lifted by the presence of the weak periodic potential, whose energy gap is compatible to the size of the potential 
(a)
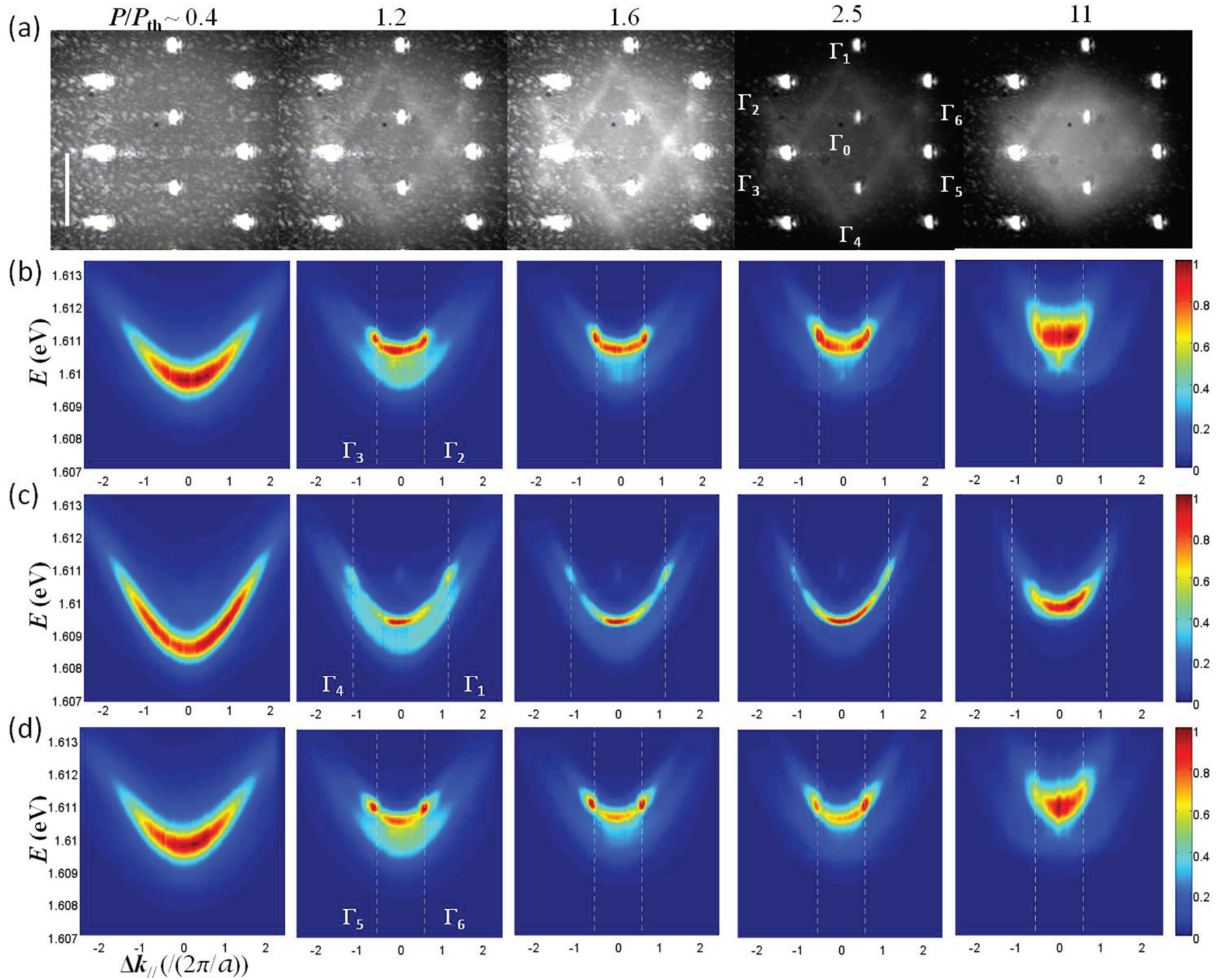

FIG. 2. (Color online) (a) Time- and energy-integrated exciton-polariton population distributions from the triangular lattice device are measured for the normalized laser pump-power values, $P / P_{\text {th }} \sim 0.4,1.2,1.6,2.5,11$ (left to right), where the threshold pump power $P_{\text {th }}$ is $3.4 \mathrm{~mW}$. The bright sharp peaks with triangular lattices come from the scattered laser signals off the lattice surface, whose distance between two nearest-neighbor peaks is the size of the reciprocal unit vectors $(2 \pi / a) 2 / \sqrt{3}$ with $a=4 \mu \mathrm{m}$. The reason of the weaker signal at $P / P_{\text {th }} \sim 2.5$ than that at $P / P_{\text {th }} \sim 1.6$ is the addition of a neutral filter for avoiding the saturation of the camera. Pump-power-dependent energy-momentum relations are presented along the three cross-sections (b) $\Gamma_{2}-\Gamma_{3}$, (c) $\Gamma_{1}-\Gamma_{4}$, and (d) $\Gamma_{5}-\Gamma_{6}$. The dotted lines are located at the values of $\Gamma_{i}$ points indicated in Fig. 1(c). The data in (b)-(d) are also taken at the same pump-power values of $P / P_{\text {th }}$. At each frame in (b)-(d), the signals are normalized by the maximum peak, which sets 1 (red) in the color scale bars.

strength, around 10-100 $\mu \mathrm{eV}$. Since both the triangular and the honeycomb lattices share common geometric symmetry, they show qualitatively similar band structures: the six degenerate states at $\Gamma$ points are reorganized into a $s$ singlet, degenerate $p$ doublet, degenerate $d$ doublet, and a $f$ singlet corresponding to real-space orbital symmetry for both lattices; the top three states would feature local momentum traps, where excitonpolaritons can reside in to establish metastable condensation.

The first observation of exciton-polariton condensates at higher bands of the $\Gamma$ points is in the momentum space images [see Figs. 2(a) and 3(a)] by varying the laser pumppower values. These images are taken over time- and energyintegration. Below threshold $\left(P / P_{\text {th }} \sim 0.4\right.$, where $P_{\text {th }}$ is the threshold pump-power value), the featureless weak ring- shaped population distribution comes from thermal excitonpolaritons within the momentum range $\left|k_{/ /}\right|<3 \times 10^{4} \mathrm{~cm}^{-1}$ (Bottleneck effect). Once quantum degeneracy threshold is reached, coherent exciton-polariton interference patterns develop at the zone boundaries near third BZ and the first BZ. The signals near the six $\Gamma_{i}(i=1-6)$ points become pronounced and sharper, forming coherent states. For the higher particle density $\left(P / P_{\text {th }} \sim 11\right)$, exciton-polaritons are eventually scattered into the lower bands [see Figs. 2(a) and 3(a), rightmost].

The density-dependent features in the energy-integrated images are further analyzed through the energy-resolved spectroscopy measurements. The particular vertical crosssections along $\Gamma_{i}$ are presented in Figs. 2(b)-2(d) and 3(b)-3(f) 


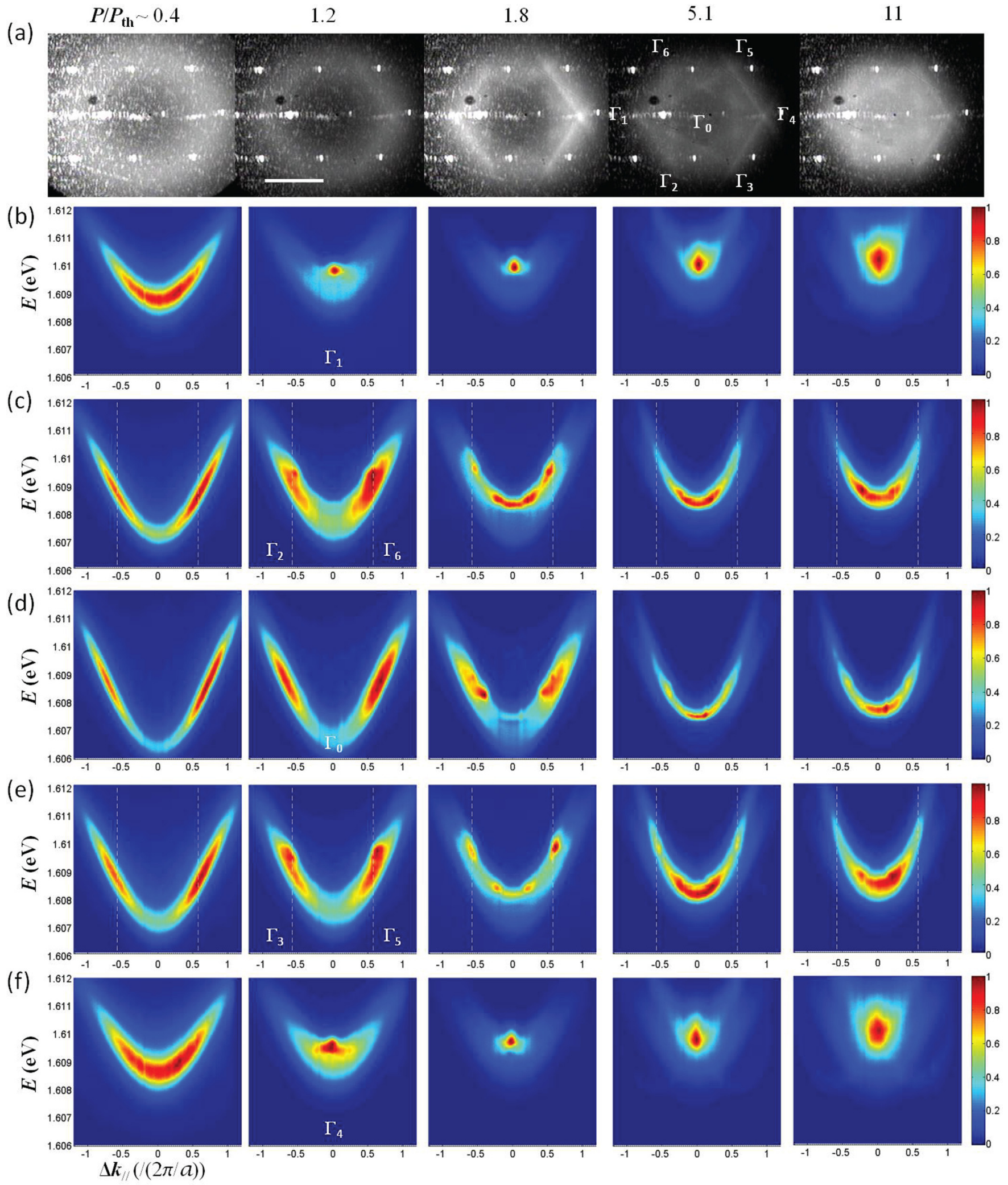

FIG. 3. (Color online) (a) Time- and energy-integrated exciton-polariton population distributions from the honeycomb lattice device as a function of the laser pump-power values, $P / P_{\text {th }} \sim 0.4,1.2,1.8,5.1,11$ (left to right), where the threshold pump power $P_{\text {th }}$ is $5.1 \mathrm{~mW}$. The bright sharp peaks caused by the laser signals calibrates the size of the reciprocal unit vectors $(2 \pi / a) 2 / 3$ with $a=2 \mu \mathrm{m}$. Pump-power dependent energy-momentum relations are presented along the five cross-sections (b) $\Gamma_{1}$, (c) $\Gamma_{2}-\Gamma_{6}$, (d) $\Gamma_{0}$, (e) $\Gamma_{3}-\Gamma_{5}$, and (f) $\Gamma_{4}$ taken at $P / P_{\text {th }} \sim 0.4$, 1.2, 1.8, 5.1, 11 (left to right). The dotted lines are located at the values of $\Gamma_{i}$ points indicated in Fig. 1(d). At each frame in (b)-(f), the signals are normalized by the maximum peak, which sets 1 (red) in the color scale bars. 
(a)

(b)
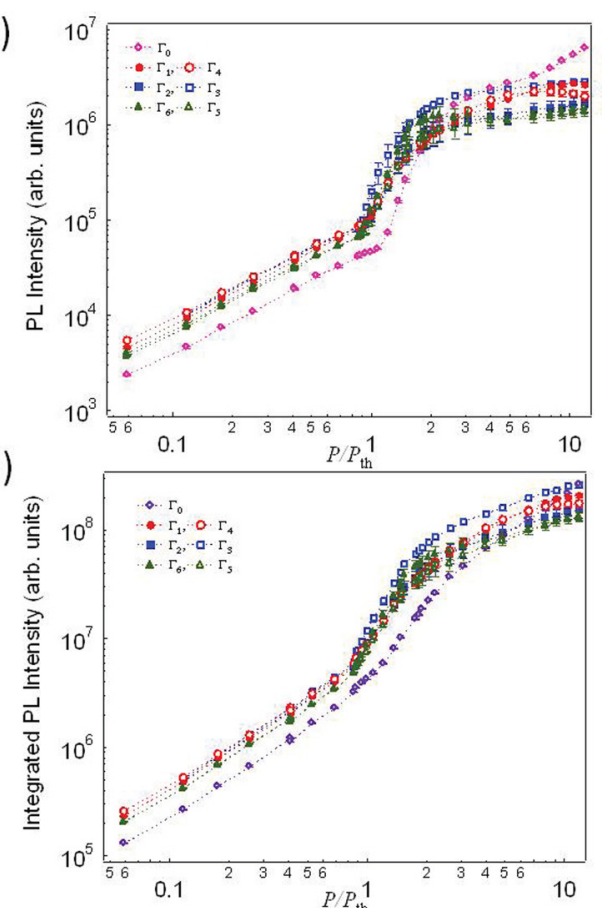

(c)

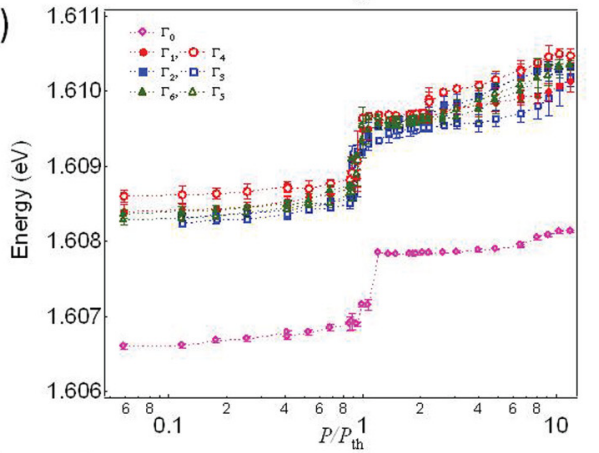

(d) $20 \times 1$

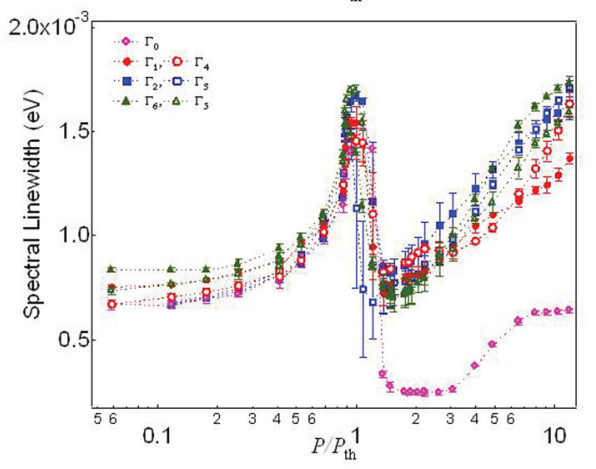

(e)

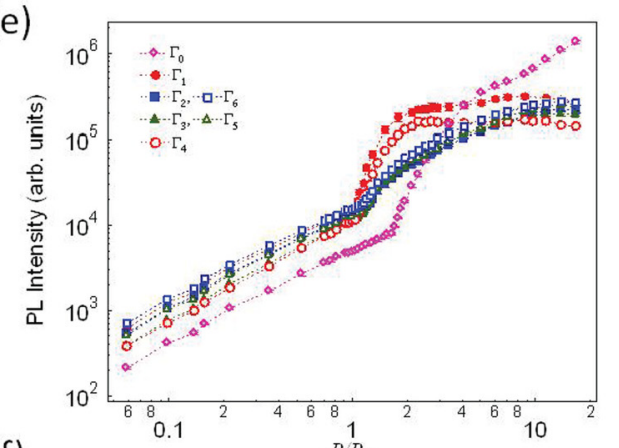

(f)

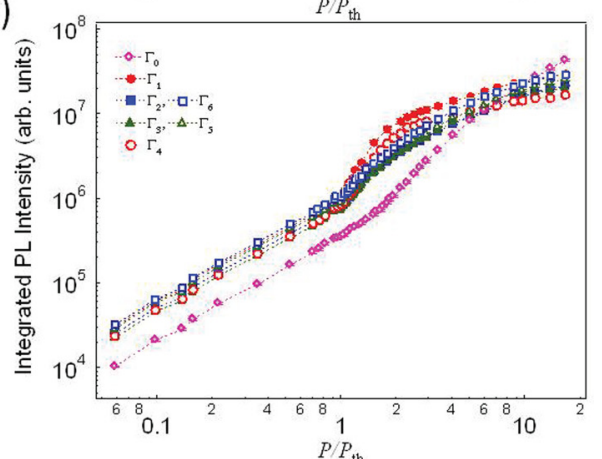

(g)

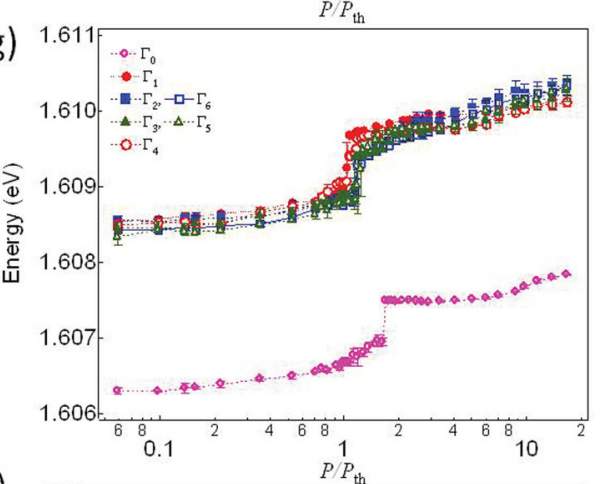

(h)

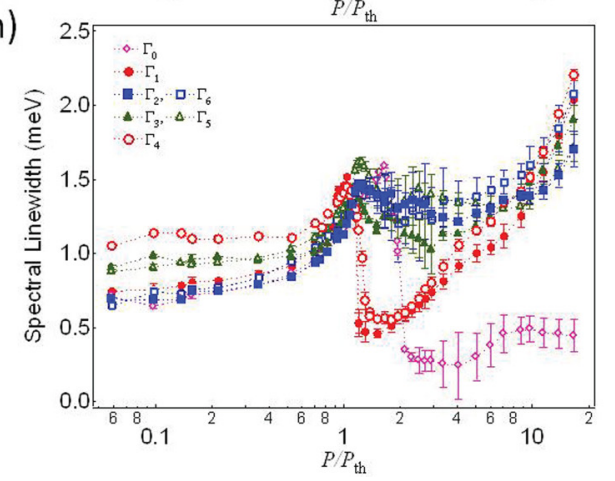

FIG. 4. (Color online) Pump-power dependent characteristics of exciton-polariton condensates at $\Gamma$ points are shown for four quantities: peak photoluminescence (PL) intensity, integrated PL intensity, the energy values, and the spectral linewidth. The data in the left column (a)-(d) are from the triangular lattice and those in the right column (e)-(h) are from the honeycomb lattices. Normalized pump-power values by the threshold value $P_{\text {th }}$ are used in $x$ axis, where $P_{\text {th }} \sim 3.4 \mathrm{~mW}$ (left) and $5.1 \mathrm{~mW}$ (right).

at various normalized pump-power values. The $x$-axis values of the spectroscopic data in Figs. 2(b)-2(d) and 3(b)-3(f) are in the unit of $2 \pi / a$. Consistent with the observation from power-dependent momentum images, below threshold, usual parabolic LP energy dispersions in all cross-sections are seen associated with the weak circular LP distribution at $P / P_{\mathrm{th}} \sim$ 0.4 in Figs. 2(a) and 3(a). Above threshold sharp Bragg peaks emerge at nonzero $\Gamma$ points in all cross-sections, and their energy values for all $\Gamma$ point signals are degenerate within the spectral linewidth $\sim 0.5 \mathrm{meV}$. As the injected polariton density increases, the $\Gamma_{i}(i=1-6)$ states experience the energy blueshift and the broadened linewidth induced by the particle-particle interactions. At high pump-power values, the Bragg peaks at the nonzero $\Gamma$ points are getting weaker and smearing into the lower momentum states, whereas the signals inside the first $\mathrm{BZ}$ are getting stronger. 

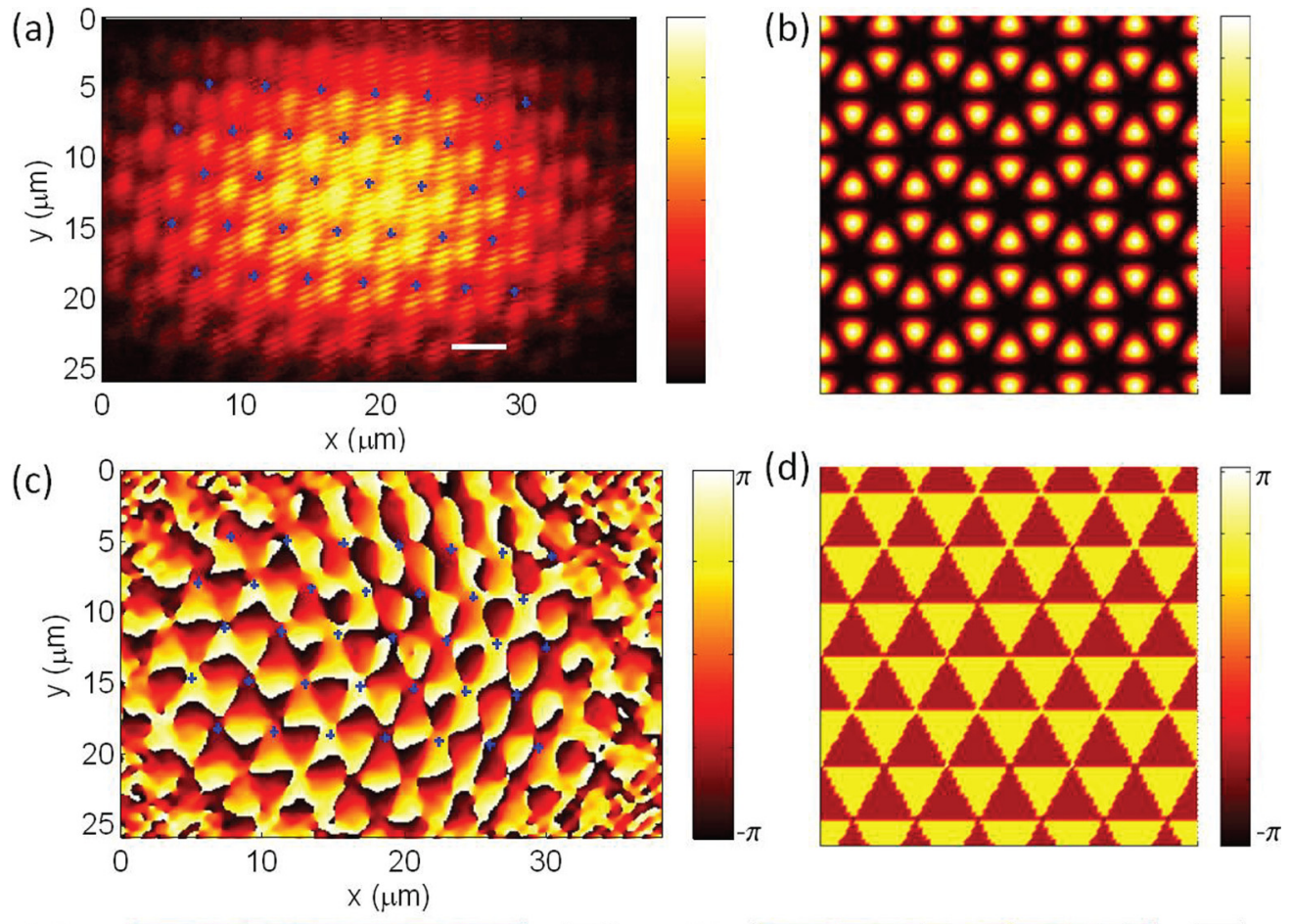

(e)

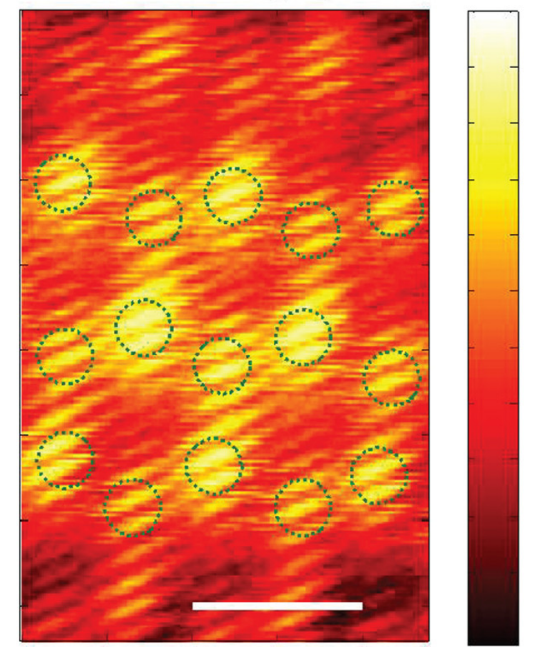

(f)

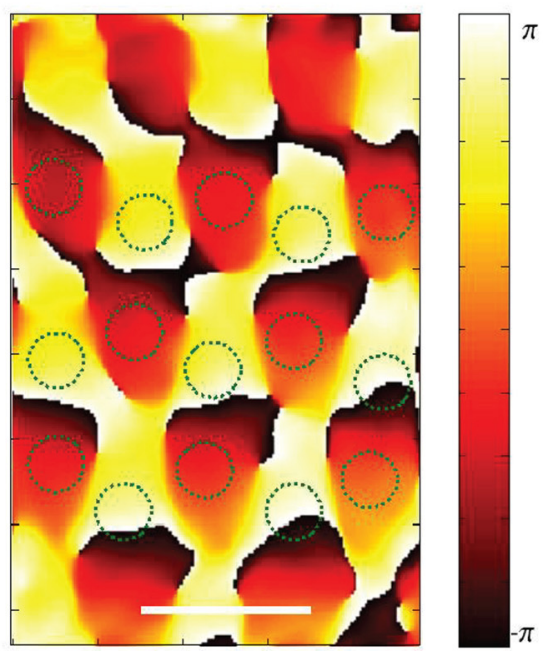

FIG. 5. (Color online) (a) Experimental real-space interferogram image in the triangular device measured at $P / P_{\text {th }} \sim 1.8$ with the normalized colorbar scale (white $=1$ and black $=0$ ). The green crosses are placed at the position of the holes. (c) The extracted phase distribution in real space in unit of $\pi$ by the off-axis interferogram analysis. Theoretical intensity distribution (b) and the phase map (d) of the $f$-singlet state at $\Gamma$ points. Zoomed-in view of the interference (e) and the phase map (f) around a few unit cells. The sixfold lobes associated with $f$-orbital symmetry are guided by dotted circles, and the white bars in images draw the length of $4 \mu \mathrm{m}$.

Figure 4 collects pump-power dependent behavior of exciton-polaritons at $\Gamma$ points extracted from Figs. 2(b)-2(d) and $3(\mathrm{~b})-3(\mathrm{f})$ in terms of four quantities: polariton peak intensity at $\Gamma_{i}(i=0-6)$ [see Fig. 4(a) for the triangular and Fig. 4(e) for the honeycomb lattices], polariton population from the integrated photoluminescence intensity [Figs. 4(b) and 4(f)], associated energy shift [Figs. 4(c) and 4(g)], and spectral linewidth [Figs. 4(d) and 4(h)]. Here, we also include the data at $\Gamma_{0}$ (pink) in comparison with those at six nonzero $\Gamma_{i}$ points with $i=1-6$. A threshold characteristic in all four quantities from both lattices allows us to define the threshold pump-power values $P_{\text {th }}$ of nonzero $\Gamma$ points to be 3.4 and $5.1 \mathrm{~mW}$ in the triangular and the honeycomb lattices, respectively. The threshold at $\Gamma_{0}$ occurs at slightly higher values. In other words, exciton-polaritons at $\Gamma_{0}$ are accumulated at higher particle density values owing to the aforementioned competition between the lifetime and the relaxation time.

Both the peak and the integrated polariton intensities at all seven $\Gamma$ points exhibit nonlinear increase near threshold pump. For $P / P_{\mathrm{th}}>4$, the polariton population at $\Gamma_{0}$ outgrows those at nonzero $\Gamma$ points, which become saturated [see Figs. 4(a) and 4(e)]. This implies that the influx rate into the global ground state at $\Gamma_{0}$ increases as the particles are further injected; however, the influx rate to nonzero $\Gamma$ points becomes balanced by the relaxation rate into the $\Gamma_{0}$ state, exhibiting the overall 


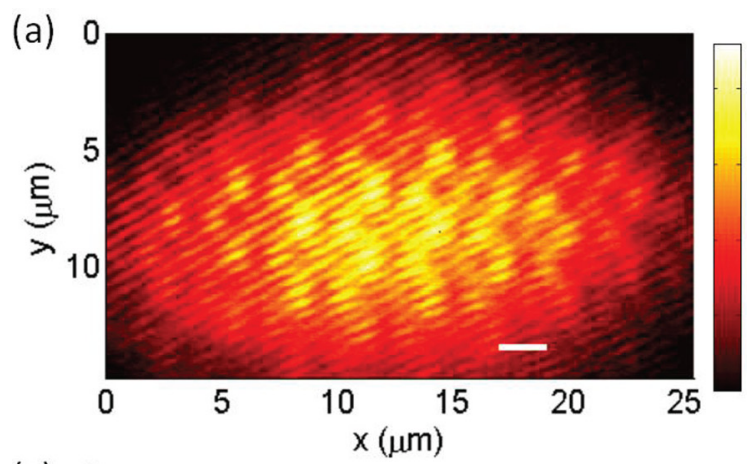

(b)
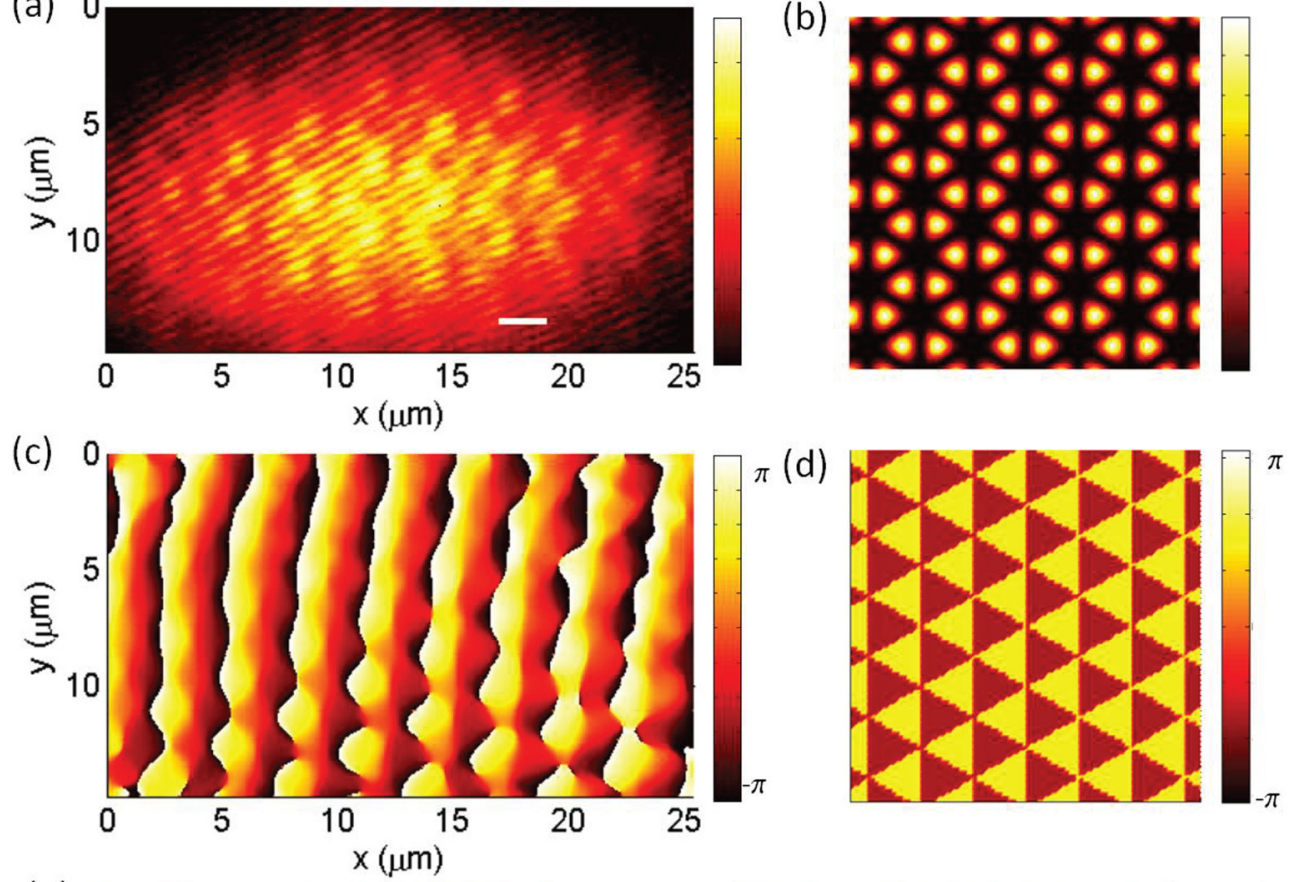

(e)

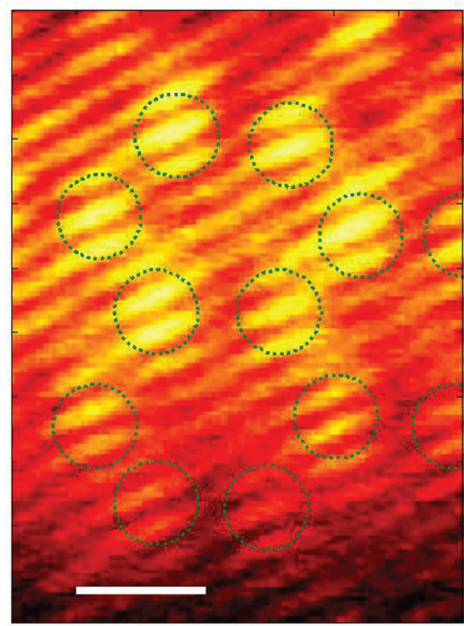

(f)

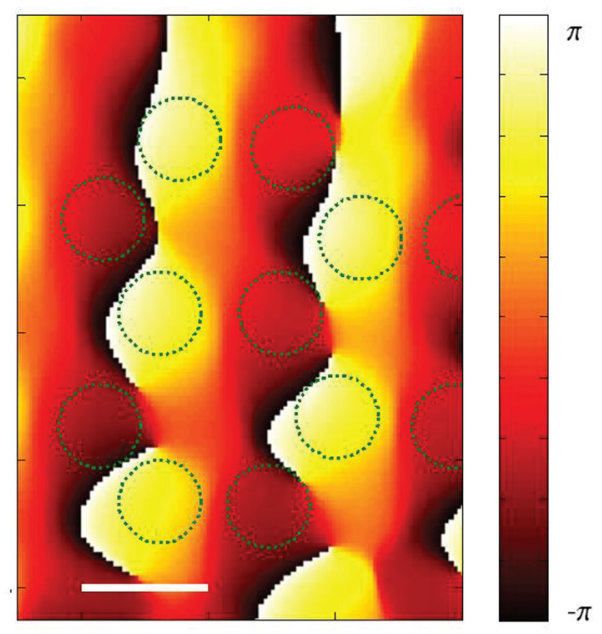

FIG. 6. (Color online) (a) Experimental real-space interferogram image in the honeycomb device measured at $P / P_{\text {th }} \sim 1.5$ with the normalized colorbar scale (white $=1$ and black $=0$ ). (c) The extracted phase distribution in real space in unit of $\pi$. Theoretical intensity distribution (b) and the phase map (d) of the $f$-singlet state at $\Gamma$ points. Zoomed-in view of the interference (e) and the phase map (f) around a few unit cells. The sixfold lobes associated with $f$-orbital symmetry are guided by dotted circles, and the white bars in images draw the length of $2 \mu \mathrm{m}$.

saturation behavior. Associated continuous energy blue shift and the spectral linewidth property (reduction near threshold and broadening above threshold) share typical signatures of exciton-polariton condensate at the zero momentum ground state in a pulsed, side pumping scheme [18,19]. We notice that the power-dependent behaviors at six nonzero $\Gamma$ points in the triangular lattice are almost identical, but those in the honeycomb lattice show some difference. Specifically, the linewidth trends at $\Gamma_{1}$ and $\Gamma_{4}$ in Fig. 4(h) are more distinctive across threshold than those at the rest of the nonzero $\Gamma$ points. At the moment, we do not have a clue to explain the discrepancy seen in the honeycomb device. Lastly, we note that the nonzero momentum metastable coherent states remain in the strong coupling regime at high pump-power values supported by the following observations: (1) less than
$2 \mathrm{meV}$ overall energy shift at all $\Gamma$ points, whose energy values are much lower than the uncoupled cavity photon energy by $\sim 5 \mathrm{meV}$; (2) the heavier effective mass of the first BZ region at high pump powers than the cavity photon mass.

\section{C. $f$-orbital symmetry of $\boldsymbol{\Gamma}$ point condensation}

In order to identify a system complex order parameter at nonzero $\Gamma$ points in real space, we construct the modified Mach-Zehnder interferometer [27] and perform a homodyne detection for a phase-resolved imaging in the energy states of nonzero $\Gamma$ points. We first eliminate the laser signal and other state signals with a narrow (1-nm band width) band pass filter. In a single arm of the interferometer, all six $\Gamma$ signals at the third BZ are chosen, and in a reference arm 
one of $\Gamma$ signals is selected by a $150-\mu \mathrm{m}$ pinhole. The six lobes associated with a $4 f_{y^{3}-3 x^{2} y}$-like orbital symmetry appear in the time-integrated interferogram above threshold shown in Figs. 5(a) and 6(a). Adopting the numerical analysis of off-axis filtering by keeping the interference components and removing the plane wave components [28], we are able to extract the underlying real-space phase distribution presented in Figs. 5(c) and 6(c). Theoretical $f$-state phase distributions in both lattices display alternating pattern between positive and negative values among six lobes [see Figs. 5(d) and 6(d)], and the relative phase difference between two values is $\pi$, analogous to the $4 f_{y^{3}-3 x^{2} y}$-like orbital symmetry appearing in the strong potential case. Figures 5(e), 5(f), 6(e), and 6 (f) are the magnified views of the clear $f$-orbital phase regions. The six lobes of $f$-orbital in experimental data are guided by dotted circles both in the raw interferogram images [Figs. 5(e) and 6(e)] and the extracted spatial phase distributions [Figs. 5(f) and 6(f)]. Except the phase polarity choice, which does not carry any physical meaning since the relative phase difference between the lobes matters, the experimental phase maps confirm that the coherent states at nonzero $\Gamma$ points carry $f$-orbital symmetry in both lattice devices. We also comment the large area maps of the phase distribution in Figs. 5(c) and 6(c) in which there are regions whose phase distributions are different from the theoretical $f$-orbital phase maps in Figs. 5(d) and 6(d). We suspect that the imperfect filtering to isolate the $f$-orbital states at nonzero $\Gamma$ points may include the contribution of the doublet $d$ states, which are separated in energy from the $f$-state energy by tens of $\mu \mathrm{eV}$. This residual $d$-state contribution may tilt and smear the sixfold phase alternation.

\section{CONCLUSION}

We identify the $f$-band exciton-polariton condensation in the 2D hexagonal lattices with the triangular and the honeycomb geometries at red-detuned devices. The direct measurements of the real-space distribution together with the momentum space distributions provide evidence of the $f$-wave orbital symmetry. Incorporating this work with addressing spin degrees of freedom by light polarization, we hope to make the polariton-lattice system as a promising platform for studying magnetic phase nature in frustrated lattices.

\section{ACKNOWLEDGMENTS}

We acknowledge Navy/SPAWAR Grant N66001-09-12024, the Japan Society for the Promotion of Science (JSPS) through its "Funding Program for World-Leading Innovative R\&D on Science and Technology (FIRST Program)," and the State of Bavaria.
[1] I. Bloch, J. Dalibard, and S. Nascimbene, Nat. Phys. 8, 267 (2012).

[2] M. Greiner, O. Mandel, T. Esslinger, T. W. Hansch, and I. Bloch, Nature (London) 415, 39 (2002).

[3] R. P. Feynman, Statistical Mechanics: A Set of Lectures (Addison-Wesley, Boston, 1972).

[4] C. Wu, Mod. Phys. Lett. B 23, 1 (2009).

[5] Y. Tokura and N. Nagaosa, Science 288, 462 (2000).

[6] A. Isacsson and S. M. Girvin, Phys. Rev. A 72, 053604 (2005).

[7] W. V. Liu and C. Wu, Phys. Rev. A 74, 013607 (2006).

[8] C. Wu, W. V. Liu, J. Moore, and S. Das Sarma, Phys. Rev. Lett. 97, 190406 (2006).

[9] C. Wu and S. Das Sarma, Phys. Rev. B 77, 235107 (2008).

[10] T. Müller, S. Fölling, A. Widera, and I. Bloch, Phys. Rev. Lett. 99, 200405 (2007).

[11] G. Wirth, M. Ölschläger, and A. Hemmerich, Nat. Phys. 7, 147 (2011).

[12] M. Ölschläger, G. Wirth, and A. Hemmerich, Phys. Rev. Lett. 106, 015302 (2011).

[13] H. Deng, H. Haug, and Y. Yamamoto, Rev. Mod. Phys. 82, 1489 (2010).

[14] E. A. Cerda-Méndez, D. N. Krizhanovskii, M. Wouters, R. Bradley, K. Biermann, K. Guda, R. Hey, P. V. Santos,
D. Sarkar, and M. S. Skolnick, Phys. Rev. Lett. 105, 116402 (2010).

[15] E. Wertz et al., Nat. Phys. 6, 860 (2010).

[16] T. Jacqmin et al., arXiv:1310.8105.

[17] N. Y. Kim et al., Phys. Stat. Solidi B 245, 1076 (2008).

[18] C. W. Lai et al., Nature (London) 450, 529 (2007).

[19] N. Y. Kim et al., Nat. Phys. 7, 681 (2011).

[20] T. Hotta, Rep. Prog. Phys. 69, 2061 (2006).

[21] C. Weisbuch, M. Nishioka, A. Ishikawa, and Y. Arakawa, Phys. Rev. Lett. 69, 3314 (1992).

[22] H. Deng et al., Science 298, 199 (2002).

[23] J. Kasprzak et al., Nature (London) 443, 409 (2006).

[24] R. Balili et al., Science 316, 1007 (2007).

[25] N. W. Ashcroft and. D. Mermin, Solid State Physics (Brooks Cole, New York, 1989).

[26] D. W. Snoke, Solid State Physics:Essential Concepts (AddisonWesley, San Francisco, 2009).

[27] K. Kusudo, N. Y. Kim, A. Löffler, S. Höfling, A. Forchel, and Y. Yamamoto, Phys. Rev. B 87, 214503 (2013).

[28] G. Nardin, Y. Léger, B. Pietka, F. Morier-Genoud, and B. Deveaud-Plédran, Phys. Rev. B 82, 045304 (2010). 